\title{
Tumour Lysis Syndrome Management in Paediatric Oncology Patients in a Single Institution. Rigorous Supportive Care Improves Cost Effectiveness \& decreases Investigative Burden in Childhood Cancer
}

\author{
Katy Walshe RN NPC, Kate Vandenberg RN NP, Peter Downie MD \\ Children's Cancer Centre, Monash Children's Hospital, Clayton Melbourne, Australia.
}

\section{Introduction}

Tumour Lysis Syndrome (TLS) is the most common paediatric oncology emergency. International guidelines suggest an approach that risk stratifies patients according to disease and laboratory values, including renal function, serum electrolytes, urate, and LDH at diagnosis.

\section{Aims}

- To examine TLS institutional practice with benchmarking against international guidelines, and review TLS supportive care needs in children diagnosed with leukaemia and lymphoma.

- To determine practice changes which would enable improved supportive care and a reduction in health-care burden and costs at the time of diagnosis.

\section{Methods}

- Single centre, observational retrospective study.

- TLS management for at-risk patients was reviewed from January 2013-December 2017.

- Records were analysed from the point of clinical presentation to first discharge from hospital.

- At-risk population defined as all children presenting with acute leukaemia or non-Hodgkin lymphoma.

- At diagnosis; analysis of clinical burden of disease, total white cell count, urate, renal function, $\mathrm{Ca}^{++}, \mathrm{PO}_{4}, \mathrm{~K}^{+}$, and $\mathrm{LDH}$.

- Subsequent days; fluid management, frequency of TLS monitoring by blood tests, and use of recombinant urate oxidase (RUO) vs. allopurinol.

\begin{tabular}{|c|c|c|c|c|c|c|}
\hline Disease & $N=105$ & $\begin{array}{l}\text { WCC } \\
<50^{*}\end{array}$ & $\begin{array}{c}\text { WCC } \\
>50<150^{* *}\end{array}$ & $\begin{array}{c}\text { WCC } \\
>150^{\#}\end{array}$ & $\begin{array}{l}\text { LDH }^{\wedge} \\
>3 x \\
\text { ULN }\end{array}$ & $\begin{array}{l}\text { Urate } \\
>2 x \\
\text { ULN }\end{array}$ \\
\hline Precursor B ALL & 65 & 60 & 4 & 1 & 0 & 3 \\
\hline T-cell ALL & 14 & 3 & 3 & 8 & 7 & 8 \\
\hline AML & 7 & 6 & 1 & 0 & 0 & 0 \\
\hline $\begin{array}{l}\text { Burkitt lymphoma } \\
\text { st III-IV }\end{array}$ & 5 & $N / A$ & N/A & N/A & 4 & 4 \\
\hline T cell NHL & 5 & $N / A$ & $N / A$ & N/A & 3 & 0 \\
\hline Burkitt leukaemia & 4 & 4 & 0 & 0 & 4 & 4 \\
\hline $\begin{array}{l}\text { Burkitt lymphoma } \\
\text { st II }\end{array}$ & 1 & N/A & $N / A$ & N/A & 0 & 0 \\
\hline $\mathrm{ALCL}$ & 3 & $N / A$ & $\mathrm{~N} / \mathrm{A}$ & N/A & 0 & 0 \\
\hline
\end{tabular}

\section{Results}

Pre B ALL;

3/65 patients met either clinical or laboratory TLS.

T cell ALL;

8/14 met TLS criteria. 9/14 had LDH>3x ULN and urate>2xULN.

$8 / 14$ had $w c c>50 \times 10^{9} / \mathrm{L}$. Those with $w c c<50 \times 10^{9} / \mathrm{L}$ did not develop TLS.

Burkitt leukaemia and lymphoma (III and IV);

$7 / 9$ patients met LTLS criteria either at diagnosis or within 24 hours of starting chemotherapy.

Treatment with RUO;

22 patients received RUO. 3/22 did not meet criteria for LTLS either at diagnosis or subsequently. $8 / 22$ received a single dose.

Of the remaining 14, 5 received 2 doses, 7 received 3 doses, and 2 patients received 4 and 5 doses respectively.

8 patients received subsequent doses RUO without meeting LTLS criteria.

\section{TLS monitoring;}

Pre B ALL; $61 / 65$ had TLS bloods done at least 8 hourly for a mean of 4 days with no change in clinical outcome.

Of the remaining 40 patients, all those with Burkitt leukaemia (4/40), Burkitt lymphoma III/IV 5/40), and T cell ALL with wCC $>50 \times 10^{9} / \mathrm{L}(8 / 40)$ met the criteria for LTLS and were appropriately monitored.

For the 73 patients who were stratified as low risk, multiple extra blood tests were performed without demonstrated change in management or outcome.

\section{Summary \& Conclusions}

Clinical assessment and analysis of laboratory criteria is critical to risk stratify patients for TLS. Laboratory TLS occurred in approximately $20 \%$.

Reliable prediction was based on; wcc $>50 \times 10^{9} / \mathrm{L}$, $\mathrm{LDH}>3 \mathrm{xULN}$ and urate $>2 \mathrm{xULN}$.

In $96 \%$ there was no change in parameters to indicate clinical concern. The majority of low risk newly diagnosed children had excess blood tests resulting in increased health care costs and patient distress. In addition, inappropriate use of UAO contributed to a significant and avoidable increase in health care expenditure.

New institutional procedures have been written based on these findings. 